\title{
Computation of Clinical Efficacy and Post- Thrombolytic Effects of Streptokinase in ST-Segment Elevated Myocardial Infarction Patients
}

\author{
Vedha Gandhirajan*, Asha K Rajan, Vedha Pal Jeyamani S*, Prakash R, Prasanth D, \\ Praveen Kumar A
}

Department of Pharmacy Practice, Jaya College of Paramedical Sciences, College of Pharmacy, Thiruninravur, Chennai, Tamil Nadu, INDIA.

\begin{abstract}
Rationale: Atherosclerosis is a prominent underlying factor to Diabetes and Hypertension seen in $80 \%$ of patients leading to increased formation of thrombus thus blocking blood supply to myocardium. Fibrinolytics, such as streptokinase in the dose of $1,500,000 \mathrm{U}$ in slow IV infusion has good activity for reperfusion. The study is aimed to evaluate the clinical efficacy and post-thrombolytic effects of streptokinase in STEMI patients. Materials and Methods: A prospective observational study of 100 patients of both sexes with STEMI was included based on inclusion and exclusion criteria. Demographic details of the patient, including ECG, Numerical Pain Rating Scale pain scale before and after fibrinolytic therapy were collected to record pain. Statistical significance was carried out with Students $t$-test, Chi- square test and SPSS software. Results: A marked change in ST segment elevation was observed with streptokinase therapy and decrease in coronary pain was measured when comparing before and after fibrinolytic therapy. At 90 min post thrombolytic therapy, 32 patients had pain relief (complete + partial), 11 patients had ST segment settlement (complete + partial) and 0 patients has $T$ wave inversion. At the end of $48 \mathrm{hr}$ of post thrombolytic therapy $>75 \%$ had pain relief, $\geq 50 \%$ patients had ST segment settlement and $\leq 10 \%$ patients had T wave inversion which was the least marker of reperfusion. Conclusion: Significant activity of streptokinase was obtained when used within $12 \mathrm{hr}$ of resolution period. Chest pain, ST segment variations were the prominent markers for testing the efficacy of therapy.
\end{abstract}

Key words: Acute coronary syndrome, Myocardial infarction, Streptokinase, Thrombolysis, ST segment elevation.

\section{INTRODUCTION}

ACS (Acute Coronary Syndrome) form the major part of cardiovascular deaths broadly classified into STEMI (ST elevated Myocardial Infarction) and NSTEMI (Non ST elevated Myocardial Infarction). STEMI results in complete occlusion of epicardial artery paving either PCI (Precutaneous Interventions) or fibrinolysis to be the effective therapy. ${ }^{1-3}$ In conditions of emergency where it becomes difficult to perform PCI, fibrinolysis becomes the immediate solution to such STEMI patients preventing early deaths. Among the other fibrinolytics, Streptokinase is a non-fibrin selective fibrinolytic involved as a thrombus and lysis, to restore supply to epicardial artery. ${ }^{4,5}$ One in five middle aged adults are known to have an underlying
CAD which could progress to MI. Most developing countries like India are expected to experience a sharp rise in Ischemic Heart Disease next to Infectious diseases. Initiation of fibrinolytic therapy immediately within some 0-3 hr brings down the short and long term complications by $15 \%$ and $25 \%$ respectively, but there is a hesitant in prescribing a fibrinolytic agent immediately. Unsuccessful reperfusion therapy with fibrinolytics could lead to its increased adverse effects causing complications. Thus time factor remains essential for success in reperfusion therapy. This reperfusion therapy with fibrinolytics not only compromises flow to epicardial artery but also enables microvascular flow monitored clearly with the help of ECG and not alone with cardio
DOI: 10.5530/ijopp.13.2.29

Address for correspondence: Prof. Vedha Pal Jeyamani.S Department of Pharmacy Practice, Jaya College of Paramedical sciences, College of Pharmacy, Thiruninravur, Chennai-602024, Tamil Nadu, INDIA.

Phone no: +91-8870114010 Email Id: swetha21112000@ gmail.com

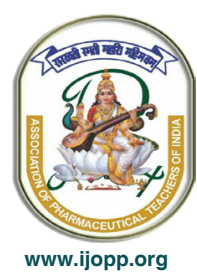


angiogram. $180 \mathrm{~min}$ is the expected time for Streptokinase rescue intervention. ${ }^{6-8}$

Clinical outcomes of fibrinolytic therapy would include resolution of elevated ST segment, relief from coronary pain, early Creatinine Kinase rise (CK-MB), development of reperfusion arrhythmias, T wave inversion from ECG. Minimum of $24 \mathrm{hr}$ is required for $\mathrm{T}$ wave inversion and $12 \mathrm{hr}$ for CK-MB peak. ${ }^{9}$ Complete resolution could be defined as the reduction in $>70 \%$, partial resolution as the reduction of $30 \%$ to $70 \%$ and no resolution as reduction of $<30 \%$ after $180 \mathrm{~min}$ of post thrombolysis in ST. PCI remains superior to fibrinolytic reperfusion but cost issues and other logistic reasons makes thrombolysis more effective and first priority. Management of MI in a developing country is still sparse due to non-availability of many resources, thus requiring strong primary prevention programs at the community level. ${ }^{10-12}$ This study is carried out with the intension of evaluating the clinical efficacy and post-thrombolytic effects of Streptokinase in STEMI patients. ${ }^{13-15}$

\section{MATERIALS AND METHODS}

\section{Duration and Design of study}

This was a prospective observational study conducted in the Cardiac Care Unit of a Tertiary care hospital in Tiruvallur, Tamil Nadu for a period of 3 months from February 2019-May 2019. The subjects included patients who were admitted during this time period in the hospital. Based upon the inclusion and exclusion criterion 100 patients of both sex with acute STEMI and further thrombolysed with Streptokinase, were included in the study. The study protocol is shown in Figure 1. Patients who were presented with chest pain, age more than or equal to 30 years and ECG with clear ST elevated MI were included. Patient's thrombolysed with any other fibrinolytics, severe cardiac failure, systolic blood pressure $<90 \mathrm{mmHg}$, past Q wave MI, acute MI within the previous 1 week, allergy to any medications, contraindications to Streptokinase within the last 6 months, any surgery or cerebrovascular accident, warfarin therapy, bleeding disorders, uncontrolled hypertension, were not included in the study.

Self-prepared proforma containing details of risk factors of CAD (Hypertension, Diabetes Melitus, Alcohol, Smoking, Family history), demographic details of the patients, laboratorial information on blood parameters, ECG variations were included. ECG was performed before and $90 \mathrm{~min}$ after fibrinolytic therapy. Pain was recorded with NPRS (Numerical pain rating scale) before and 90 min after fibrinolytic therapy. Informed consent and Institutional Ethics Clearance were obtained before initiation of the study.

\section{Statistical analysis}

Data was analyzed with the help of SPSS software after the arrangement in Microsoft Excel sheet. Qualitative data was analyzed with the help of Chi-square test, Continuous data was analyzed with its Mean and Standard deviations. A $p$ value of $<0.05$ was considered statistically significant.

\section{RESULTS}

Among the total of 100 STEMI patients included in the study $62(62 \%)$ were Male and $38(38 \%)$ were Female

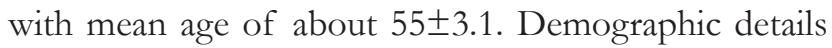
of patients are elaborated in Table $1.63 \%$ of STEMI patients were diabetic, 55\% were with Hypertensive status and $44 \%$ with dyslipidemia. This shows how much threat patients face with cardiovascular conditions. About $37 \%$ of patients presented with Anterior MI, 30\% with Lateral MI, 20\% with Inferior MI and 13\% with Inferior + Right ventricular MI as shown in Table 2.

About 18 (12.7\%) were suspected with family history, $29(20.5 \%)$ with smoking, 20 (14.17\%) with alcohol, 31 $(21.9 \%)$ with hypertension, $28(19.8 \%)$ with Diabetes and $15(10.6 \%)$ with obesity as risk factors for STEMI episode as elaborated in Table 3. Table 4 has clearly depicted the association between pain perception before streptokinase administration and $90 \mathrm{~min}$ after streptokinase administration with the risk factors. Table 5 shows association between ST segment elevation before streptokinase administration and $90 \mathrm{~min}$ after streptokinase administration with the risk factors. Demography of the patients with reperfusion is depicted in Table 6.

At 90 min post thrombolytic therapy, 32 patients has pain relief (complete + partial), 11 patients has ST segment settlement (complete + partial) and 0 patients has $\mathrm{T}$ wave inversion. At the end of $48 \mathrm{hr}$ of post thrombolytic therapy $>75 \%$ had pain relief, $\geq 50 \%$ patients had ST segment settlement and $\leq 10 \%$ patients had $\mathrm{T}$ wave inversion which is the least marker of reperfusion. Table 7 shows the comparison on ECG resolution with risk factor variables with complete (46), partial (28) and failed (26) therapy.

\section{DISCUSSION}

Among the fibrinolytics for reperfusion therapy, Streptokinase is the most commonly employed one and is already stated in other studies for its efficacy among other fibrinolytics, reduced stroke and hemorrhagic 
Table 1: Demographic details of patients presenting with STEMI.

\begin{tabular}{ccc}
\hline Parameters & $\begin{array}{c}\text { Frequency } \\
(\boldsymbol{n}=\mathbf{1 0 0})\end{array}$ & Percentage (\%) \\
\hline Gender & 38 & 38 \\
Female & 62 & 62 \\
Male & & \\
Smoking & 42 & 42 \\
Non-smoker & 36 & 36 \\
Current smoker & 22 & 22 \\
Ex-smoker & & \\
Diabetic status & 63 & 63 \\
Yes & 37 & 37 \\
No & & \\
Exercise habit & 43 & 43 \\
Yes & 57 & 57 \\
No & & 55 \\
Hypertension level & 55 & 45 \\
Yes & 45 & 44 \\
No & & 66 \\
Hyperlipidemia status & 44 & \\
Yes & 66 & \\
No & &
\end{tabular}

Table 2: Site of infarction on ECG and its changes.

\begin{tabular}{ccc} 
Parameters & $\begin{array}{c}\text { Frequency } \\
(\boldsymbol{n = 1 0 0 )}\end{array}$ & Percentage (\%) \\
\hline Anterior MI (V1-V6) & 37 & 37 \\
Lateral MI (I, aVL, V5,V6) & 30 & 30 \\
Inferior MI (II, III, aVF) & 20 & 20 \\
Inferior + Rt Ventricular MI & 13 & 13 \\
(II, III, aVF + V4R) & & \\
\hline
\end{tabular}

\section{Table 3: Risk factors of CAD.}

\begin{tabular}{ccc}
\hline Risk Parameters & Frequency & Percentage (\%) \\
\hline Family History & 18 & 12.7 \\
Smoking & 29 & 20.5 \\
Alcohol & 20 & 14.17 \\
Hypertension & 31 & 21.9 \\
Diabetes & 28 & 19.8 \\
Obesity & 15 & 10.6 \\
\hline
\end{tabular}

complications, feasibility, safety and decreased mortality rates. The resolution time in widow period was high in $0-4 \mathrm{~h}$ which is not significant with that of $\leq 12 \mathrm{hr}$ as per other studies. Thus, frequency of thrombolysis and baseline risk factors has no connectivity with the resolution time. ${ }^{16,17}$

A statistical significance of $(p<0.001)$ was obtained in the NPRS pain scale before administration and $90 \mathrm{~min}$ after initiating streptokinase therapy (Figure 2). Similarly a statistical value of $p \leq 0.005$ was obtained with ST segment resolution and streptokinase therapy. Schroder et al. have mentioned in his studies that resolution of ST segment from initiation of therapy would give a clear picture of clinical efficacy. Studies state that hypertension is one of the prevalent factors for MI occurrence. ${ }^{18,19}$

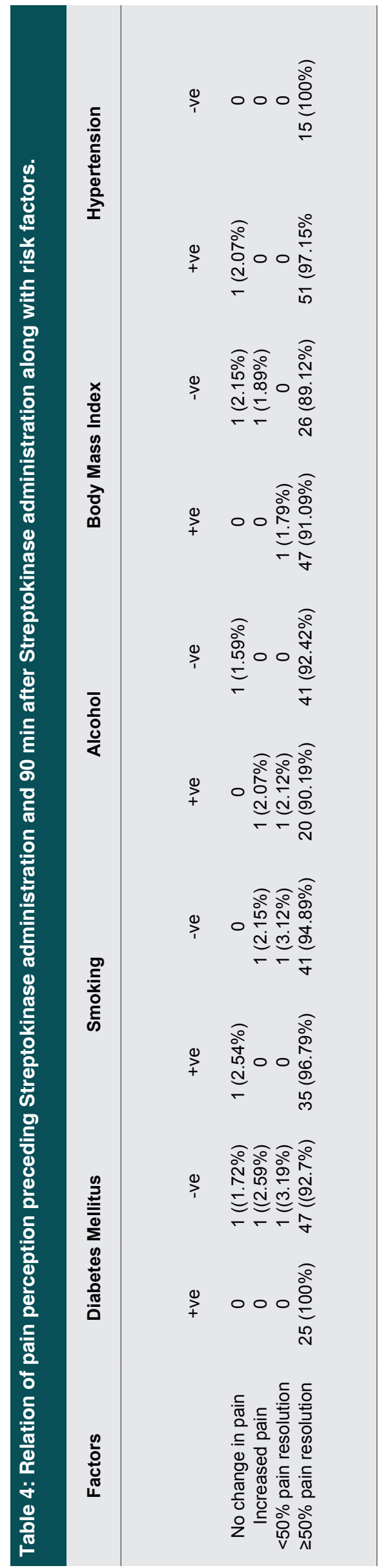


Table 5: Relation of ST segment elevation preceding Streptokinase administration and 90 min after Streptokinase administration with risk factors.

\begin{tabular}{|c|c|c|c|c|c|c|c|c|c|c|}
\hline \multirow[t]{2}{*}{ Factors } & \multicolumn{2}{|c|}{ Diabetes Mellitus } & \multicolumn{2}{|c|}{ Smoking } & \multicolumn{2}{|c|}{ Alcohol } & \multicolumn{2}{|c|}{ Body Mass Index } & \multicolumn{2}{|c|}{ Hypertension } \\
\hline & + ve & -ve & + ve & -ve & $+v e$ & -ve & + ve & -ve & $+v e$ & -ve \\
\hline $\begin{array}{l}\text { Increased ST } \\
\text { segment }\end{array}$ & $\begin{array}{c}3 \\
(8.01 \%)\end{array}$ & $\begin{array}{c}5 \\
(13.39 \%\end{array}$ & $\begin{array}{c}1 \\
(15.70 \%)\end{array}$ & $\begin{array}{c}3 \\
(3.21 \%)\end{array}$ & $\begin{array}{c}5 \\
(5.19 \%)\end{array}$ & $\begin{array}{c}2 \\
(8.19 \%)\end{array}$ & $\begin{array}{c}4 \\
(8.16 \%)\end{array}$ & $\begin{array}{c}7 \\
(8.19 \%)\end{array}$ & $\begin{array}{c}9 \\
(25.75 \%)\end{array}$ & $\begin{array}{c}5 \\
(37.20 \%)\end{array}$ \\
\hline$<50 \%$ ST & 3 & 13 & 7 & 13 & 9 & 15 & 13 & 12 & 7 & 2 \\
\hline $\begin{array}{l}\text { segment } \\
\text { resolution }\end{array}$ & $(15.21 \%)$ & $(25.19 \%)$ & $(10.27 \%)$ & $(20.70 \%)$ & $(18.35 \%)$ & $(18.19 \%)$ & $(6.19 \%)$ & $(21.23 \%)$ & $(21.23 \%)$ & $(15.79 \%)$ \\
\hline $\begin{array}{l}\geq 50 \% \text { ST } \\
\text { segment } \\
\text { resolution }\end{array}$ & $\begin{array}{c}25 \\
(77.19 \%)\end{array}$ & $\begin{array}{c}33 \\
(67.09 \%)\end{array}$ & $\begin{array}{c}27 \\
(72.52 \%)\end{array}$ & $\begin{array}{c}28 \\
(70.25 \%)\end{array}$ & $\begin{array}{c}20 \\
(80.19 \%)\end{array}$ & $\begin{array}{c}29 \\
(70.50 \%)\end{array}$ & $\begin{array}{c}19 \\
(75.15 \%)\end{array}$ & $\begin{array}{c}38 \\
(66.90 \%)\end{array}$ & $\begin{array}{c}45 \\
(79.21 \%)\end{array}$ & $\begin{array}{c}10 \\
(59.19 \%)\end{array}$ \\
\hline
\end{tabular}

\begin{tabular}{|c|c|c|c|c|c|}
\hline \multirow{2}{*}{$\begin{array}{c}\text { Time period } \\
\text { (After } \\
\text { initiating } \\
\text { thrombolysis) }\end{array}$} & \multicolumn{2}{|c|}{ Pain relief } & \multicolumn{2}{|c|}{$\begin{array}{l}\text { ST segment } \\
\text { settlement }\end{array}$} & \multirow[t]{2}{*}{$\begin{array}{c}\text { T wave } \\
\text { inversion }\end{array}$} \\
\hline & Complete & Partial & Complete & Partial & \\
\hline $15 \min$ & - & - & - & - & - \\
\hline $30 \mathrm{~min}$ & 1 & 1 & 3 & 1 & - \\
\hline $1 \mathrm{hr}$ & 12 & 10 & 3 & 1 & - \\
\hline $1: 30 \mathrm{hr}$ & 3 & 5 & 2 & 1 & - \\
\hline $2 \mathrm{hr}$ & 9 & 3 & 8 & 2 & 1 \\
\hline $3 \mathrm{hr}$ & 7 & 5 & 4 & 3 & 3 \\
\hline 3 to $24 \mathrm{hr}$ & 9 & 4 & 3 & 3 & 5 \\
\hline 24 to $48 \mathrm{hr}$ & 6 & 1 & 1 & 2 & 1 \\
\hline
\end{tabular}

Table 7: Demography of comparison on ECG resolution with other variables.

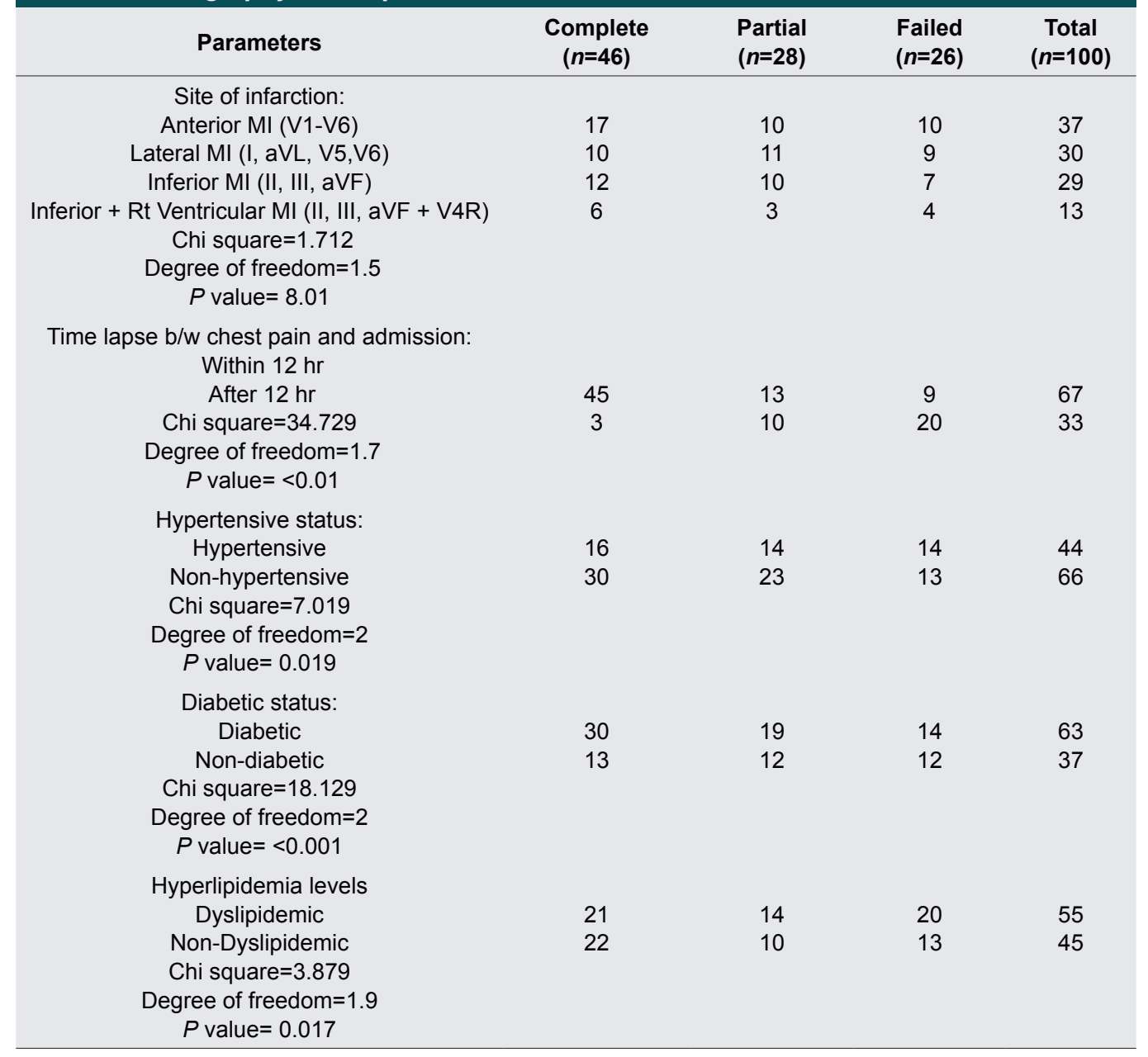




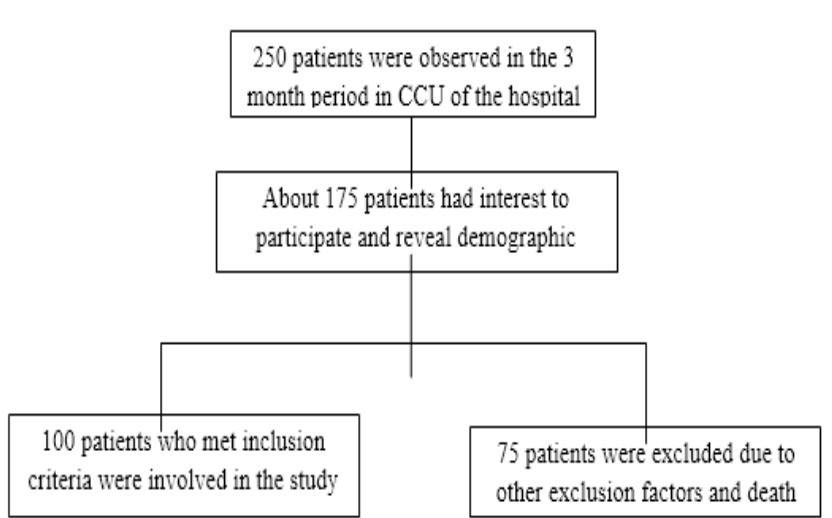

Figure 1: Study protocol.

In our study too, hypertensive patients were presented with higher ST segment elevation when compared to nonhypertensive patients. (Yun Lin, Weiqi Pan et al.) Krinsley $\mathrm{Js}$ and Fischer $\mathrm{M}$ reported the same change in diabetic patients too which is in accordance with our study.

Alcoholic and smoking groups were found to be less benefitted with thrombolytic therapy when compared to the other groups. This again poses major threat and risks. 67 patients received therapy within $12 \mathrm{hr}$ of pain where 45 patients had complete resolution, the others had predisposed factor of Diabetes, Hypertension to cause partial or failed resolution. A $p$ value of $<0.01$ was observed. Relation between Dyslipidemia and resolution time was not much evident with our study unlike diabetes and hypertension. ${ }^{20,21}$

Among the other risk factors age becomes a nonmodifiable cause of STEMI. The minimum age was 32 years and a maximum of 77 years. Female patients were found with low incidence of STEMI when compared to male population, especially women belonging to premenopausal stage. This was in accordance with other studies by Misiriya KI et al. and Hananio G et al..$^{15,7,9}$ Finally the study reported a mortality of about $7 \%$ of patients which was inevitable. Hence it was highly recommended to control risk factors towards STEMI like smoking, alcohol, obesity, Diabetes and Hypertension. Hospital setup where primary PCI is not immediately accessible could switch-on to fibrinolytic reperfusions immediately within $12 \mathrm{hr}$.

\section{Limitations}

The study limits with a small sample size and its observational nature which may compromise with some of its results. The variation with results is possible due to admitted patients between hospitals and their arrival. Comparison among other fibrinolytic agents was not done due to common use of streptokinase in the hospital when compared to other fibrinolytics.

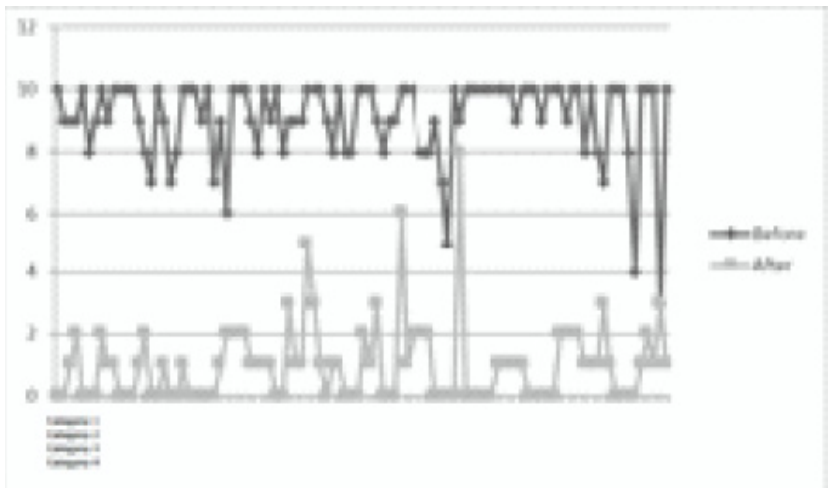

Figure 2: Individual patient value indicating the pain score before and after administration of Streptokinase in STEMI patients.

\section{CONCLUSION}

The study details the importance of immediate fibrinolytic therapy in case of difficulty in PCI, due to its reduced cost, complications and mortality rates. Post thrombolytic effects of Streptokinase within $12 \mathrm{hr}$ of resolution are highly efficacies stating its importance. Risk factors like smoking and alcohol possess threat for both cardiovascular conditions and poor drug therapeutic effects, which has to be controlled.

\section{ACKNOWLEDGEMENT}

We thank the patients and their caretakers in giving consent for participation in this study. In addition we thank the health care professionals who helped in carrying out the work successfully.

\section{CONFLICT OF INTEREST}

The authors declare no conflict of interest.

\section{ABBREVIATIONS}

ACS: Acute coronary syndrome; CAD: Coronary artery disease; CCU: Cardiac care unit; CK-MB: Creatinine kinase-muscle brain; NPRS: Numerical pain rating scale; PCI: Percutaneous Coronary Intervention; STEMI: ST segment elevated myocardial infarction.

\section{SUMMARY}

The research summarizes that streptokinase is an effective fibrinolytic agent and an immediate first line in STEMI patients when compared to PCI which imposes high cost and time consuming.

\section{REFERENCES}

1. Gupta MC, Mehta L, Gupta SP. Clinical profile of acute myocardial infarction with special reference to risk factors-a five-year study. JAPI. 1989;37(1):55.

Indian Journal of Pharmacy Practice, Vol 13, Issue 2, Apr-Jun, 2020 
2. Dwivedi S, Anupam P, Chaturvedi A. Cardiovascular risk factors in young coronary heart disease patients around East Delhi. South Asian J Prevent Cardiol. 1997;1:21-6.

3. Khan S, Abrar A, Abid AR, Jan T, Khan H. In hospital outcome of patients having acute myocardial infarction with and without streptokinase. Gomal J Med Sci. 2009;7(2):31.

4. Schroder R. Prognostic impact of early ST-segment resolution in acute ST elevation myocardial infarction. Circulation. 2004;110(21):506-10.

5. DeLemos JA, Braunwald E. ST segment resolution as a tool for assessing the efficacy of reperfusion therapy. J Am Coll Cardiol. 2001;38(5):1283-94.

6. DeWerf FV, Ardissino D, Betriu A, Cokkinos DV, Falk E, Fox KA, et al. Management of acute myocardial infarction in patients presenting with STsegment elevation. European Heart J. 2003;24(1):28-66.

7. Jajoo U, Taksande B, Yelwatkar S, Kumar R. Resolution of coronary pain and settlement of elevated ST Segment after streptokinase thrombolysis in STEMI as a marker of prognosis. JMGIMS. 2011;16(1):60-3.

8. Anuj RV, Swapnil C, Kamble TK, Sourya A. Clinical Markers of Reperfusion in Patients with Acute Myocardial Infarction and its Prognosis Significance. International Journal of Recent Surgicals and Medical Sciences. 2016;2(2):905.

9. Rolf S, Rüdiger D, Thomas B, Karl W, Thomas L, Ulrich T, et al. Extent of early ST segment elevation resolution: A simple but strong predictor of outcome in patients with acute myocardial infarction. Journal of the American College of Cardiology. 1994;24(2):384-91.

10. James AL, Eugene B. ST segment resolution as a tool for assessing the efficacy of reperfusion therapy. Journal of the American College of Cardiology. 2001;38(5):1283-94.

11. Nazif A, Kurthuluş Ö, Adnan A, Meryem UA, Mehmet AD. Mehmet AV, et al. Prevalence of risk factors of ST segment elevation myocardial infarction in Turkish patients living in Central Anatolia. The Anatolian Journal of Cardiology. 2009;9(1):3-8

12. Ragupathy A, Nanda KK, Hira P, Hassan K, Oscar HF, DiAngelantonio $\mathrm{E}$, et al. Hypertension in India: A systematic review and meta-analysis of prevalence, awareness and control of hypertension. Journal of Hypertension. 2014;32(6):1170-7.

13. Yun L, Weiqi P, Shangqiu N, Xianto S, Zening J, Shuzheng L. Prevalence and management of hypertension in patients with acute coronary syndrome vary with gender: Observations from Chinese Registry of Acute Coronary Events (CRACE). Molecular Medicine Reports. 2013;8(1):173-7.

14. James SK, Molly F. The diabetic paradox: Diabetes is not independently associated with mortality in critically ill patients. Hospital Practice. 2012;40(2):315.

15. Javed IM, Azhar M, Tariq JM, Ifaat T. Study on ST Segment Elevation Acute Myocardial Infarction in Diabetic and Non-Diabetic Patients. Pakistan Journal of Medical Sciences. 2008;24(6):786-91.

16. Johanson P, Jernberg T, Gunnarsson G, Lindahl B, Wallentin L, Dellborg M. Prognostic value of ST-segment resolution-when and what to measure. Eur Heart J. 2003;24(4):337-45.

17. Mustafa T, Yuling F, Galen S, Shaun G, Christopher B, Lars W, et al. Risk stratification in ST elevation myocardial infarction is enhanced by combining baseline ST deviation and subsequent ST segment resolution. Heart. 2008;94(3):e6.

18. Aly $\mathrm{MH}$, Bader A, et al. Early Changes in Ventricular Repolarization after Thrombolytic Therapy in Patients with Acute Myocardial Infarction as Indicators for Prediction of Epicardial Coronary Artery Reperfusion. International Journal of Kuwait University of Health Sciences. 2007;16(2):19-20.

19. Schröder R, Zeymer U, Wegscheider K, Neuhaus KL. Comparison of the predictive value of ST segment elevation resolution at 90 and $180 \mathrm{~min}$ after start of streptokinase in acute myocardial infarction: A substudy of the Hirudin for improvement of Thrombolysis (HIT)-4 study. Eur Heart J. 1999;20(21):1563-71.

20. James A, David AM, Gibson CM, et al. Early noninvasive detection of failed epicardial reperfusion after fibrinolytic therapy. American Journal of Cardiology. 2001;88(4):353-8.

21. Ophuis A, Bar F, Vermeer F, Janssen W, Doevendans P, Haest R. Angiographic assessment of prospectively determined noninvasive reperfusion indices in acute myocardial infarction. Heart. 2000;84(2):164-70. 